\title{
GORENSTEIN AND $S_{r}$ PATH IDEALS OF CYCLES
}

\author{
DARIUSH KIANI \\ Department of Pure Mathematics, Faculty of Mathematics and Computer Science, Amirkabir University of \\ Technology (Tehran Polytechnic), 424, Hafez Ave., Tehran 15914, Iran, and School of Mathematics, \\ Institute for Research in Fundamental Sciences (IPM), Tehran, Iran \\ e-mail:dkiani@aut.ac.ir,dkiani7@gmail.com \\ SARA SAEEDI MADANI \\ e-mail: sarasaeedi@aut.ac.ir \\ and NAOKI TERAI \\ Naoki Terai, Department of Mathematics, Faculty of Culture and Education, \\ Saga University, Saga 840-8502, Japan \\ e-mail: terai@cc.saga-u.ac.jp
}

(Received 23 July 2013; accepted 7 September 2013; first published online 26 August 2014)

\begin{abstract}
Let $R=k\left[x_{1}, \ldots, x_{n}\right]$, where $k$ is a field. The path ideal (of length $t \geq 2$ ) of a directed graph $G$ is the monomial ideal, denoted by $I_{t}(G)$, whose generators correspond to the directed paths of length $t$ in $G$. Let $C_{n}$ be an n-cycle. We show that $R / I_{t}\left(C_{n}\right)$ is $S_{r}$ if and only if it is Cohen-Macaulay or $\left\lceil\frac{n}{n-t-1}\right\rceil \geq r+3$. In addition, we prove that $R / I_{t}\left(C_{n}\right)$ is Gorenstein if and only if $n=t$ or $2 t+1$. Also, we determine all ordinary and symbolic powers of $I_{t}\left(C_{n}\right)$ which are Cohen-Macaulay. Finally, we prove that $I_{t}\left(C_{n}\right)$ has a linear resolution if and only if $t \geq n-2$.

2010 Mathematics Subject Classification. 13D02, 13F55, 05C75, 05 C38.
\end{abstract}

1. Introduction. Let $G=(V, E)$ be a finite simple graph with vertex set $V=$ $\left\{x_{1}, \ldots, x_{n}\right\}$ and edge set $E$. Associated to $G$ is a monomial ideal

$$
I(G)=\left(x_{i} x_{j}:\left\{x_{i}, x_{j}\right\} \in E\right)
$$

in the polynomial ring $R=k\left[x_{1}, \ldots, x_{n}\right]$ over a field $k$, called the edge ideal of $G$.

The path ideal of a graph was first introduced by Conca and De Negri in [4]. Fix an integer $n \geq t \geq 2$ and let $G$ be a directed graph. A sequence $x_{i_{1}}, \ldots, x_{i_{t}}$ of distinct vertices is called the path of length $t$ if there are $t-1$ distinct directed edges $e_{1}, \ldots, e_{t-1}$, where $e_{j}$ is a directed edge from $x_{i_{j}}$ to $x_{i_{j+1}}$. Then the path ideal of $G$ of length $t$ is the monomial ideal

$$
I_{t}(G)=\left(x_{i_{1}} \cdots x_{i_{t}}: x_{i_{1}}, \ldots, x_{i_{t}} \text { is the path of length } t \text { in } G\right) .
$$

Clearly, $I_{2}(G)=I(G)$, thus the path ideal is also called the generalised edge ideal of $G$. It is shown in [4] that the Rees algebra $\mathcal{R}\left(I_{t}(G)\right)$ is normal and Cohen-Macaulay when $G$ is a directed tree. In [12], it is determined when the path ideal of a cycle is sequentially Cohen-Macaulay. Also, in [11], all trees whose path ideals are unmixed, Cohen-Macaulay and Gorenstein are characterised. 
In this paper, we study some properties of the path ideals of cycles. Throughout the paper, we mean by $C_{n}$ the $n$-cycle with directed edges $e_{1}, \ldots, e_{n}$, where $e_{i}$ is from $x_{i}$ to $x_{i+1}$ for $i=1, \ldots, n-1$ and $e_{n}$ is from $x_{n}$ to $x_{1}$. Moreover, we have $I_{t}\left(C_{n}\right)=\left(u_{1}, \ldots, u_{n}\right)$, where $u_{i}=\prod_{v=0}^{t-1} x_{i+v}$ for all $i=1, \ldots, n$. Note that here the indices are considered in $\mathbb{Z}_{n}$. In [7], the authors dealt with the case of $t=2$ and determined when $R / I_{2}\left(C_{n}\right)$ satisfies Serre's condition $S_{r}$ and sequentially $S_{r}$. Also, it is well known that $n=5$ is the only case in which $R / I_{2}\left(C_{n}\right)$ is Gorenstein. Here we consider all $t>2$ and study Gorenstein, $S_{r}$ and sequentially $S_{r}$ properties of $R / I_{t}\left(C_{n}\right)$ in general.

This paper is organised as follows. In the next section, we recall several definitions and terminology which we need later. In Section 2, we show that for $t \geq 3, R / I_{t}\left(C_{n}\right)$ is $S_{r}$ if and only if it is Cohen-Macaulay or $\left\lceil\frac{n}{n-t-1}\right\rceil \geq r+3$. Actually, we show that just in these cases, the minimal graded free resolution of the Alexander dual of the path ideal of a cycle is linear in the first $r$ steps. In Section 3, we prove that for $t \geq 3$, $R / I_{t}\left(C_{n}\right)$ is Gorenstein if and only if $n=t$ or $2 t+1$. To prove this, we investigate when the last non-vanishing Betti number of $R / I_{t}\left(C_{n}\right)$ is 1 . Moreover, we identify the powers of the path ideal of a cycle which are Cohen-Macaulay. In Section 4, we prove that $I_{t}\left(C_{n}\right)$ has a linear resolution if and only if $t \geq n-2$.

2. Preliminaries. Let $\Delta$ be a simplicial complex, and let $F_{1}, \ldots, F_{q}$ be all the facets of $\Delta$. We sometimes write $\Delta=\left\langle F_{1}, \ldots, F_{q}\right\rangle$. Now we define the simplicial complex $\Delta_{t}(G)$ to be

$$
\Delta_{t}(G)=\left\langle\left\{v_{i_{1}}, \ldots, v_{i_{t}}\right\}: v_{i_{1}}, \ldots, v_{i_{t}} \text { is a path of length } \mathrm{t} \text { in } G\right\rangle,
$$

where $G$ is a directed graph. A vertex cover of $\Delta$ is a subset $A$ of $V$, with the property that for every facet $F_{i}$ there is a vertex $x_{j} \in A$ such that $x_{j} \in F_{i}$. A minimal vertex cover of $\Delta$ is a subset $A$ of $V$ such that $A$ is a vertex cover and no proper subset of $A$ is a vertex cover of $\Delta$.

Now supppose that $\Delta$ is a simplicial complex of dimension $d-1$. Let $f_{i}=f_{i}(\Delta)$ denote the number of faces of dimension $i$. Sequence $f(\Delta)=\left(f_{0}, f_{1}, \ldots, f_{d-1}\right)$ is called the $f$-vector of $\Delta$. Letting $f_{-1}=1$, the $h$-vector $h(\Delta)=\left(h_{0}, h_{1}, \ldots, h_{d}\right)$ of $\Delta$ is defined by the formula

$$
\sum_{i=0}^{d} f_{i-1}(t-1)^{d-i}=\sum_{i=0}^{d} h_{i} t^{d-i} .
$$

Moreover, we have the following well-known theorem.

THEOREM 2.1 [14, Theorem 5.4.6]. Let $\Delta$ be a simplicial complex. If $h(\Delta)=$ $\left(h_{0}, h_{1}, \ldots, h_{d}\right)$ and $f(\Delta)=\left(f_{0}, f_{1}, \ldots, f_{d-1}\right)$ are $f$-vector and $h$-vector of $\Delta$, respectively, then

$$
h_{j}=\sum_{i=0}^{j}(-1)^{i} f_{j-i-1}\left(\begin{array}{c}
d-j+i \\
i
\end{array}\right) \text {, }
$$

where $d=\operatorname{dim}(k[\Delta])$.

We say that a pure $(d-1)$-dimensional simplicial complex $\Delta$ is strongly connected if for any two facets $F$ and $G$, there exists a sequence of facets $F=F_{0}, F_{1}, \ldots, F_{m}=G$ 
such that $\left|F_{i-1} \cap F_{i}\right|=d-1$ for all $i=1, \ldots, m$. The Alexander dual of $\Delta$ is the simplicial complex

$$
\Delta^{\vee}=\left\{F^{c}: F \notin \Delta\right\}
$$

Let $I$ be a squarefree monomial ideal. The squarefree Alexander dual of $I=$ $\left(x_{1,1} \cdots x_{1, s_{1}}, \ldots, x_{t, 1} \cdots x_{t, s_{t}}\right)$ is the ideal

$$
I^{\vee}=\left(x_{1,1}, \ldots, x_{1, s_{1}}\right) \cap \cdots \cap\left(x_{t, 1}, \ldots, x_{t, s_{t}}\right) .
$$

Suppose $I$ is a homogeneous ideal of $R$ whose all generators have degree $d$. Then $I$ has a $d$-linear resolution (or simply linear resolution) if for all $i \geq 0, \beta_{i, j}(I)=0$ for all $j \neq i+d$.

If $I$ is a graded ideal of $R$, then we write $I_{\langle j\rangle}$ for the ideal generated by all homogeneous polynomials of degree $j$ belonging to $I$. We say that a graded ideal $I \subset R$ is component-wise linear if $I_{\langle j\rangle}$ has a linear resolution for all $j$.

3. Serre's condition $S_{r}$ and the path ideals of $C_{n}$. In this section, we show that $R / I_{t}\left(C_{n}\right)$ is $S_{r}$ if and only if it is Cohen-Macaulay or $\left\lceil\frac{n}{n-t-1}\right\rceil \geq r+3$. Recall that a finitely generated graded module $M$ over the Noetherian-graded $k$-algebra $S$ is said to satisfy the Serre's condition $S_{r}$ if depth $M_{P} \geq \min \left(r, \operatorname{dim} M_{P}\right)$ for all $P \in \operatorname{Spec}(S)$.

The following is the main theorem of this section.

Theorem 3.1. Let $3 \leq t \leq n$ and $r \geq 2$. Then $R / I_{t}\left(C_{n}\right)$ is $S_{r}$ if and only if it is Cohen-Macaulay or $\left\lceil\frac{n}{n-t-1}\right\rceil \geq r+3$.

To prove the theorem, we need some facts which are mentioned in the sequel. In [15, Corollary 3.7], Yanagawa (with Terai) showed that a simplicial complex is $S_{r}$ if and only if the minimal free resolution of its Alexander dual is linear in the first $r$ steps, where $r \geq 2$. We will use this fact to determine when $R / I_{t}\left(C_{n}\right)$ is $S_{r}$. Also, we need some facts about a special kind of simplicial complexes, which we will use in the proof of the main theorem of this section.

A generalised tree is defined inductively as follows:

(i) A simplex is a generalised tree.

(ii) If $\Delta$ is a generalised tree with the vertex set $V$, so is $\Delta \cup \operatorname{co}_{x_{0}} F$ for any $F \in \Delta$ and for any new vertex $x_{0}$, where $\mathrm{co}_{x_{0}} F$ is the simplex on the vertex set $F \cup\left\{x_{0}\right\}$.

LEMMA 3.2 [1, Lemma 2]. For any simplicial complex $\Delta$ which is not a simplex, the Stanley-Reisner ring $K[\Delta]$ of $\Delta$ has a 2-linear resolution if and only if $\Delta$ is a generalised tree.

A pure $d$-dimensional strongly connected generalised tree is called a $d$-tree. This notion can also be characterised inductively as follows:

(i) A $d$-simplex is a $d$-tree.

(ii) If $\Delta$ is a $d$-tree, so is $\Delta \cup \mathrm{co}_{x_{0}} F$ for any $F \in \Delta$ with $|F|=d$ and any new vertex $x_{0}$.

Now we prove Theorem 3.1.

Proof of Theorem 3.1. Let $q:=\left\lceil\frac{n}{n-t-1}\right\rceil-1$. 
'Only if' Suppose that $R / I_{t}\left(C_{n}\right)$ is $S_{r}$ so that $I_{t}\left(C_{n}\right)$ is unmixed. Suppose $R / I_{t}\left(C_{n}\right)$ is not Cohen-Macaulay. So $t+2 \leq n \leq\left\lfloor\frac{3 t}{2}\right\rfloor+1$ by [12, Theorem 3.1 and Corollary 3.6]. Also, let $\left\lceil\frac{n}{n-t-1}\right\rceil<r+3$. Thus, $2 \leq q-1 \leq r$, and hence $R / I_{t}\left(C_{n}\right)$ is $S_{q-1}$. Note that the minimal generators of $\left(I_{t}\left(C_{n}\right)\right)^{\vee}$ have degree 2 (see [12, proof of Theorem 3.1]). For simplicity, we denote $\left(I_{t}\left(C_{n}\right)\right)^{\vee}$ by $I_{t}\left(C_{n}\right)^{\vee}$. So the minimal graded free resolution of $I_{t}\left(C_{n}\right)^{\vee}$ is 2-linear in the first $q-1$ steps. Thus, $\beta_{q-2, j}\left(I_{t}\left(C_{n}\right)^{\vee}\right)=0$ for all $j \neq q$. Let $\alpha:=n-t-1$ and $W:=\{\alpha, 2 \alpha, \ldots, q \alpha, n\}$. Let $\Delta$ be the Stanley-Reisner simplicial complex of $I_{t}\left(C_{n}\right)^{\vee}$ so that $I_{\Delta}=I_{t}\left(C_{n}\right)^{\vee}$. Thus, $F \subseteq V=\left\{x_{1}, \ldots, x_{n}\right\}$ is a face of $\Delta$ if and only if it does not contain any vertex covers of $\Delta_{t}\left(C_{n}\right)$. So $\Delta_{W}$ is a $(q+1)$-cycle over $W$, since just any two consecutive vertices in $W$ do not yield a vertex cover of $\Delta_{t}\left(C_{n}\right)$. Hence, $\operatorname{dim}_{k} \widetilde{H}_{1}\left(\Delta_{W} ; k\right)=1$. So, by Hochster's formula (see [9, Theorem 8.1.1]), we have $\beta_{q-2, q+1}\left(I_{t}\left(C_{n}\right)^{\vee}\right) \neq 0$, which is a contradiction. Thus, $\left\lceil\frac{n}{n-t-1}\right\rceil \geq r+3$ or $R / I_{t}\left(C_{n}\right)$ is Cohen-Macaulay.

'If' If $R / I_{t}\left(C_{n}\right)$ is Cohen-Macaulay, then it is also $S_{r}$. By [12, Corollary 3.6], one may assume that $n \geq t+2$. Now, suppose that $\left\lceil\frac{n}{n-t-1}\right\rceil \geq r+3$. Then $q-2 \geq r$. We show that $R / I_{t}\left(C_{n}\right)$ is $S_{q-2}$, and hence it is also $S_{r}$. Note that by an easy computation, one can see that $t \geq(n-1)\left(\frac{r+1}{r+2}\right)$. So $n \leq\left\lfloor\frac{3 t}{2}\right\rfloor+1$, since $r \geq 2$. Therefore, $I_{t}\left(C_{n}\right)$ is unmixed by [12, Theorem 3.1], and $I_{t}\left(C_{n}\right)^{\vee}$ is generated by elements of degree 2. Also, note that the minimal graded free resolution of $R / I_{t}\left(C_{n}\right)$ is of the form

$$
0 \rightarrow R(-n) \rightarrow R(-t-1)^{n} \rightarrow R(-t)^{n} \rightarrow R \rightarrow R / I_{t}\left(C_{n}\right) \rightarrow 0
$$

by [2, Proposition 3.3], and hence $\operatorname{pd}\left(R / I_{t}\left(C_{n}\right)\right)=\operatorname{reg}\left(I_{t}\left(C_{n}\right)^{\vee}\right)=3$. On the other hand, we have $q-3 \leq \operatorname{pd}\left(I_{t}\left(C_{n}\right)^{\vee}\right)$. Thus, it suffices to show that $\beta_{q-3, j}\left(I_{t}\left(C_{n}\right)^{\vee}\right)=0$ for all $j \neq q-1$. To prove this, it is enough to show that $\beta_{q-3, q}\left(I_{t}\left(C_{n}\right)^{\vee}\right)=0$, since $\operatorname{reg}\left(I_{t}\left(C_{n}\right)^{\vee}\right)=3$. Let $\Delta$ be the Stanley-Reisner simplicial complex of $I_{t}\left(C_{n}\right)^{\vee}$. Then $\operatorname{dim}(\Delta)=n-t-1$. Let $U \subseteq V=\left\{x_{1}, \ldots, x_{n}\right\}$ with $|U|=q$. By Hochster's formula, it suffices to show that $\operatorname{dim}_{K} \widetilde{H}_{1}\left(\Delta_{U} ; k\right)=0$. Suppose that $U=\left\{x_{i_{1}}, \ldots, x_{i_{q}}\right\}$ such that $i_{1}<\cdots<i_{q}$. Let $y_{j}$ be the number of vertices between $x_{i_{j}}$ and $x_{i_{j+1}}$ on $C_{n}$ (in the direction of $C_{n}$ ) for all $j=1, \ldots, q-1$, and $y_{q}$ be the number of vertices between $x_{i_{q}}$ and $x_{i_{1}}$. Then there exists an integer $l$ such that $1 \leq l \leq q$ and $y_{l} \geq n-t-1$. So there exists a subset $X$ of $V$ which consists of exactly $t+1$ consecutive vertices and $U \subseteq X$. Thus, it is easy to see that $\Delta_{X}$ is a $(n-t-1)$-tree. Therefore, $I_{\Delta_{X}}$ has a 2-linear resolution by Lemma 3.2. So by Hochster's formula, we have $\operatorname{dim}_{k} \widetilde{H}_{1}\left(\Delta_{U} ; k\right)=0$, since $\Delta_{U} \subseteq \Delta_{X}$. The desired result now follows.

A graded $R$-module $M$ is called sequentially $S_{r}$ (over $k$ ) if there exists a finite filtration of graded $R$-modules $0=M_{0} \subset M_{1} \subset \cdots \subset M_{r}=M$ such that each $M_{i} / M_{i-1}$ is $S_{r}$, and the Krull dimensions of the quotients are increasing, i.e.

$$
\operatorname{dim}\left(M_{1} / M_{0}\right)<\operatorname{dim}\left(M_{2} / M_{1}\right)<\cdots<\operatorname{dim}\left(M_{r} / M_{r-1}\right) .
$$

THEOREM 3.3 [7, Corollary 2.7]. Let $I$ be a squarefree monomial ideal in $R=$ $k\left[x_{1}, \ldots, x_{n}\right]$. Then $R / I$ is $S_{r}$ if and only if $R / I$ is sequentially $S_{r}$ and $I$ is unmixed.

THEOREM 3.4. Let $3 \leq t \leq n$. Then we have:

(a) For $r \geq 3, R / I_{t}\left(C_{n}\right)$ is $S_{r}$ if and only if it is sequentially $S_{r}$.

(b) Let $n=q t+\alpha$, where $\alpha=0$ or $2 \leq \alpha \leq t-1$. Then $R / I_{t}\left(C_{n}\right)$ is $S_{2}$ if and only if it is sequentially $S_{2}$. 
Proof. (a) By Theorem 3.3, it suffices to show that if $R / I_{t}\left(C_{n}\right)$ is sequentially $S_{r}$, then $I_{t}\left(C_{n}\right)$ is unmixed. It was shown in [12, proof of Theorem 4.1] that when $I_{t}\left(C_{n}\right)$ is not unmixed, $I_{t}\left(C_{n}\right)^{\vee}$ is not component-wise linear in the first three steps. So by [7, Theorem 3.2], $R / I_{t}\left(C_{n}\right)$ is not sequentially $S_{r}$.

(b) By a similar argument as in part (a), we get the result.

REMARK 3.5. In Theorem 3.4 part (b), $\alpha$ could not be 1 . For example, let $n=10$ and $t=3$. Then one can see by CoCoA that $R / I_{3}\left(C_{10}\right)$ is sequentially $S_{2}$ (over $\mathbb{Q}$ ), but not $S_{2}$, by Theorem 3.1.

4. Gorenstein path ideals of $C_{n}$ and Cohen-Macaulayness of powers of $I_{t}\left(C_{n}\right)$. In this section, we determine when $R / I_{t}\left(C_{n}\right)$ is Gorenstein. Also, we deal with all ordinary and symbolic powers of $I_{t}\left(C_{n}\right)$ which are Cohen-Macaulay.

The next theorem shows the relation between Cohen-Macaulay and Gorenstein properties of $R / I$, where $I$ is a homogeneous ideal in $R$.

THeOREM 4.1 [14, Corollary 4.3.5]. If $R$ is a polynomial ring over field $k$, then $a$ homogeneous ideal $I$ of $R$ is Gorenstein if and only if $R / I$ is Cohen-Macaulay and the last Betti number in the minimal graded resolution of $R / I$ is equal to 1 .

Also, we need the following theorem, which gives a formula for computing the Betti numbers of the Stanley-Reisner rings with linear resolutions.

THEOREM 4.2 [8, Corollary 3.2]. Let $\Delta$ be a $(d-1)$-dimensional simplicial complex. Suppose that the Stanley-Reisner ring $R / I_{\Delta}$ has an m-linear free resolution. If $h(\Delta)=$ $\left(h_{0}, \ldots, h_{d}\right)$ is the h-vector of $\Delta$, then

$$
(-1)^{i+1} \beta_{i}\left(I_{\Delta}\right)=\sum_{l=0}^{m+i}(-1)^{l} h_{m+i-l}\left(\begin{array}{c}
n-d \\
l
\end{array}\right)
$$

for each $0 \leq i \leq p$, where $p=\operatorname{pd}\left(I_{\Delta}\right)$.

Now we prove the main theorem of this section.

THEOREM 4.3. Let $t \geq 3$. Then $R / I_{t}\left(C_{n}\right)$ is Gorenstein if and only if $n=t$ or $2 t+1$.

Proof. By [12, Corollary 3.6], we show that if $n=t$ or $2 t+1$, then $R / I_{t}\left(C_{n}\right)$ is Gorenstein, but $R / I_{t}\left(C_{t+1}\right)$ is not, since we know that if $R / I_{t}\left(C_{n}\right)$ is Gorenstein, then it is Cohen-Macaulay. By Theorem 4.1, it is enough to show that the last non-vanishing Betti number of $R / I_{t}\left(C_{n}\right)$ is 1 for $n=t$ or $2 t+1$, but it is greater than 1 for $n=t+1$. Obviously, if $n=t$, then $0 \rightarrow R(-t) \rightarrow R \rightarrow R / I_{t}\left(C_{n}\right) \rightarrow 0$ is the minimal graded free resolution of $R / I_{t}\left(C_{n}\right)$. If $n=2 t+1$, then by [2, Proposition 3.3], the minimal graded free resolution of $R / I_{t}\left(C_{n}\right)$ is of the form

$$
0 \rightarrow R(-n) \rightarrow R(-t-1)^{n} \rightarrow R(-t)^{n} \rightarrow R \rightarrow R / I_{t}\left(C_{n}\right) \rightarrow 0 .
$$

Hence, when $n=t$ or $2 t+1$, the last non-vanishing Betti numbers are equal to 1 . Now, suppose that $n=t+1$. We have $\operatorname{dim}\left(R / I_{n-1}\left(C_{n}\right)\right)=n-2$. Since $R / I_{n-1}\left(C_{n}\right)$ is Cohen-Macaulay, we have $\operatorname{pd}\left(R / I_{n-1}\left(C_{n}\right)\right)=n-\operatorname{dim}\left(R / I_{n-1}\left(C_{n}\right)\right)$ by the AuslanderBuchsbaum formula. So $\operatorname{pd}\left(R / I_{n-1}\left(C_{n}\right)\right)=2$, and hence $\operatorname{pd}\left(I_{n-1}\left(C_{n}\right)\right)=1$. Now we should compute $\beta_{1}\left(I_{n-1}\left(C_{n}\right)\right)$. Note that by [5, Theorem 3], $R / I_{n-1}\left(C_{n}\right)$ has an $(n-1)$ linear resolution, since $I_{n-1}\left(C_{n}\right)=I\left(K_{n}\right)^{\vee}$ and $R / I\left(K_{n}\right)$ is Cohen-Macaulay. Let $\Delta$ 
be the Stanley-Reisner simplicial complex of $I_{n-1}\left(C_{n}\right)$. So we have $\operatorname{dim}(\Delta)=n-3$. Therefore, by Theorem 4.2, we have

$$
\begin{aligned}
\beta_{1}\left(I_{n-1}\left(C_{n}\right)\right) & =\sum_{l=0}^{n}(-1)^{l+2} h_{n-l}(\Delta)\left(\begin{array}{l}
2 \\
l
\end{array}\right) \\
& =\sum_{l=0}^{2}(-1)^{l+2} h_{n-l}(\Delta)\left(\begin{array}{l}
2 \\
l
\end{array}\right) \\
& =h_{n-2}(\Delta),
\end{aligned}
$$

since $h_{n}(\Delta)=h_{n-1}(\Delta)=0$. Now, by Theorem 2.1, we compute $h_{n-2}(\Delta)$ :

$$
\begin{aligned}
h_{n-2}(\Delta) & =\sum_{i=0}^{n-2}(-1)^{i} f_{n-i-3}(\Delta)\left(\begin{array}{l}
i \\
i
\end{array}\right) \\
& =\sum_{i=0}^{n-2}(-1)^{i} f_{n-i-3}(\Delta) \\
& =\sum_{i=0}^{n-2}(-1)^{i}\left(\begin{array}{c}
n \\
n-i-2
\end{array}\right) \\
& =n-1,
\end{aligned}
$$

since $f_{j}(\Delta)=\left(\begin{array}{c}n \\ j+1\end{array}\right)$ for all $j=0, \ldots, n-3$. Thus, $\beta_{1}\left(I_{n-1}\left(C_{n}\right)\right)=n-1>1$, as desired.

We end this section by a result on the symbolic powers of $I_{t}\left(C_{n}\right)$. Let us first recall that a matroid is a collection of subsets of a finite set, called independent sets, with the following properties:

(i) The empty set is independent.

(ii) Every subset of an independent set is independent.

(iii) If $F$ and $G$ are two independent sets and $F$ has more elements than $G$, then there exists an element in $F$ which is not in $G$ that when added to $G$ still gives an independent set.

Clearly, we may consider a matroid as a simplicial complex.

By $I_{\Delta}^{(m)}$, we denote the $m$ th symbolic power of the Stanley-Reisner ideal of a simplicial complex $\Delta$. Note that it is well known that Cohen-Macaulayness of $I_{\Delta}^{2}$ is equivalent to Cohen-Macaulayness of $I_{\Delta}^{(2)}$ and $I_{\Delta}^{2}=I_{\Delta}^{(2)}$. Now we use this fact together with Theorem 4.3 and [12, Corollary 3.6] to deduce the following.

THEOREM 4.4. Let $t \geq 3$ and $I:=I_{t}\left(C_{n}\right)$. Then we have:

(a) If $t=n$, then $I^{m}=I^{(m)}$ is Cohen-Macaulay for all $m \geq 1$.

(b) If $t=\frac{n-1}{2}$, then $I^{m}$ (resp. $I^{(m)}$ ) is Cohen-Macaulay if and only if $m \leq 2$.

(c) If $t=n-1$, then $I^{(m)}$ is Cohen-Macaulay for all $m \geq 1$, but $I^{m}$ is CohenMacaulay if and only if $m=1$. 
(d) If t is none of the above cases, then none of the powers of I is Cohen-Macaulay.

Proof. (a) and (d) are clear.

(b) Suppose that $t=\frac{n-1}{2}$. Let $m \geq 3$ and $\Delta$ be the Stanley-Reisner simplicial complex of $I$. Since $\Delta$ is not a complete intersection, $I^{m}$ is not Cohen-Macaulay by [13, Theorem 1.2]. Now set $F=\left\{x_{1}, x_{2}, \ldots, x_{t-2}, x_{t}, x_{t+1}, x_{t+2}, \ldots, x_{2 t-2}\right\}$ and $G=$ $\left\{x_{1}, x_{2}, \ldots, x_{t-1}, x_{t+1}, x_{t+2}, \ldots, x_{2 t-1}\right\}$. Note that $F$ and $G$ are faces of $\Delta$, since they do not contain any $t$ consecutive vertices of $C_{n}$. Also, we have $2 t-3=|F|<|G|=2 t-2$ and if any of the elements of $G \backslash F$ is added to $F$, then the new set does not belong to $\Delta$. So $\Delta$ is not a matroid. Thus, $I^{(m)}$ is not Cohen-Macaulay by [13, Theorem 1.1]. Now, let $m=2$. By [12, Lemma 3.2], we have $\operatorname{dim}(R / I)=2 t-2$ and hence $I$ is a Gorenstein Stanley-Reisner ideal of co-dimension 3. Thus, $I^{2}=I^{(2)}$ is Cohen-Macaulay by [10, Corollary 5.3].

(c) Let $t=n-1$ and $\Delta$ be the Stanley-Reisner simplicial complex of $I$. It is easy to see that $\Delta$ is a matroid. Therefore, $I^{(m)}$ is Cohen-Macaulay for all $m \geq 1$, by [13, Theorem 1.1]. For $m \geq 3, I^{m}$ is not Cohen-Macaulay because $I$ is not a complete intersection. Since $\Delta$ is not Gorenstein and $I^{(2)}$ is Cohen-Macaulay, $I^{2}$ is not CohenMacaulay by [10, Lemma 2.2].

5. Path ideals and linear resolutions. In this section, we identify when the path ideals of cycles have a linear resolution. First, let us introduce the notion of properly connected simplicial complexes which was defined for hypergraphs by Hà and Van Tuyl [6].

DEFINITION 5.1. Let $\Delta$ be a pure simplicial complex where every facet has dimension $d$. A Chain of length $m$ in $\Delta$ is a sequence of facets $\left(F_{0}, \ldots, F_{m}\right)$ such that (1) $F_{0}, \ldots, F_{m}$ are all distinct facets of $\Delta$, and

(2) $F_{i} \cap F_{i+1} \neq \emptyset$, for all $i=0, \ldots, m-1$.

Two facets $F$ and $F^{\prime}$ are connected if there exists a chain $\left(F_{0}, \ldots, F_{m}\right)$, where $F=F_{0}$ and $F^{\prime}=F_{m}$. The chain connecting $F$ to $F^{\prime}$ is a proper chain if $\left|F_{i} \cap F_{i+1}\right|=\left|F_{i+1}\right|-1$ for all $i=0, \ldots, n-1$. The (proper) chain is an (proper) irredundant chain of length $n$ if no proper subsequence is a (proper) chain from $F$ to $F^{\prime}$. The distance between two facets $F$ and $F^{\prime}$ in $\Delta$ is then given by

$$
\operatorname{dist}_{\Delta}\left(F, F^{\prime}\right)=\min \left\{l:\left(F=F_{0}, \ldots, F_{l}=F^{\prime}\right) \text { is a proper irredundant chain }\right\} ;
$$

if no such chain exists, then set $\operatorname{dist}_{\Delta}\left(F, F^{\prime}\right)=\infty$. We say that $\Delta$ is properly connected if

$$
\operatorname{dist}_{\Delta}\left(F, F^{\prime}\right)=(d+1)-\left|F \cap F^{\prime}\right|
$$

for any two facets $F, F^{\prime} \in \Delta$ with the property that $F \cap F^{\prime} \neq \emptyset$. Otherwise, we say that $\Delta$ is not properly connected.

The diameter of $\Delta$ is

$$
\operatorname{diam}(\Delta)=\max \left\{\operatorname{dist}_{\Delta}\left(F, F^{\prime}\right): F, F^{\prime} \in \Delta\right\},
$$

where the diameter is infinite if there exist two facets not connected by any proper chain. 
Lemma 5.2. Let $2 \leq t \leq n / 2$. Then $\Delta_{t}\left(C_{n}\right)$ is properly connected and $\operatorname{diam}\left(\Delta_{t}\left(C_{n}\right)\right)=\lfloor n / 2\rfloor$.

Proof. It is straightforward by definitions.

LEMMA 5.3. $I_{t}\left(C_{n}\right)^{\vee}=I_{\Delta_{n-t}\left(C_{n}\right)}$.

Proof. It is easy to see that $\Delta_{t}\left(C_{n}\right)=\overline{\Delta_{n-t}\left(C_{n}\right)}$. So $\left(I_{\Delta_{n-t}\left(C_{n}\right)}\right)^{\vee}=I_{\left(\Delta_{n-t}\left(C_{n}\right)\right)^{\vee}}=$ $I\left(\overline{\Delta_{n-t}\left(C_{n}\right)}\right)=I\left(\Delta_{t}\left(C_{n}\right)\right)=I_{t}\left(C_{n}\right)$, where the second equality holds by $[9$, Lemma 1.5.3].

THEOREM 5.4 [14, Theorem 5.4.8]. Let $\Delta$ be a simplicial complex of dimension $d$ and $k$ an infinite field. If $k[\Delta]$ is Cohen-Macaulay, then $h_{i}(\Delta) \geq 0$ for all $i=0, \ldots, d+1$, where $h_{i}(\Delta)$ is the ith component of the h-vector of $\Delta$.

Now we are ready to prove the main theorem of this section.

THEOREM 5.5. Let $t \geq 2$. Then $I_{t}\left(C_{n}\right)$ has a linear resolution if and only if $t \geq n-2$.

Proof. 'If' If $t=n$, then the result is clear. We know that $I_{n-1}\left(C_{n}\right)=I\left(K_{n}\right)^{\vee}$ and $I_{n-2}\left(C_{n}\right)=I\left(C_{n}^{c}\right)^{\vee}$. On the other hand, $R / I\left(K_{n}\right)$ and $R / I\left(C_{n}^{c}\right)$ are Cohen-Macaulay. Thus, by [5, Theorem 3], $I_{t}\left(C_{n}\right)$ has a linear resolution if $t \geq n-2$.

'Only If' If $2 \leq t \leq n-3$, then we show that $I_{t}\left(C_{n}\right)$ does not have a linear resolution. First, suppose that $2 \leq t \leq n / 2$. In this case if $I_{t}\left(C_{n}\right)$ has a linear resolution then obviously it has linear first syzygies. On the other hand, by Lemma 5.2, $\Delta_{t}\left(C_{n}\right)$ is properly connected. So by [6, Theorem 7.4] we get $\lfloor n / 2\rfloor \leq t$, hence $t=\lfloor n / 2\rfloor$. Now, suppose that $\lfloor n / 2\rfloor \leq t \leq n-3$, and let $t^{\prime}:=n-t$. Then $3 \leq t^{\prime} \leq n-\lfloor n / 2\rfloor$. In this case we show that $R / I_{\Delta_{t^{\prime}}\left(C_{n}\right)}$ is not Cohen-Macaulay, then by Lemma 5.3, $R / I_{t}\left(C_{n}\right)^{\vee}$ is not Cohen-Macaulay and $I_{t}\left(C_{n}\right)$ does not have a linear resolution. Note that $f_{i}\left(\Delta_{t^{\prime}}\left(C_{n}\right)\right)=n\left(\begin{array}{c}t^{\prime}-1 \\ i\end{array}\right)$ for all $i=0, \ldots, t^{\prime}-1$. So,

$$
\begin{aligned}
h_{j}\left(\Delta_{t^{\prime}}\left(C_{n}\right)\right) & =\sum_{i=0}^{j}(-1)^{i} f_{j-i-1}\left(\begin{array}{c}
t^{\prime}-j+i \\
i
\end{array}\right) \\
& =n \sum_{i=0}^{j-1}(-1)^{i}\left(\begin{array}{c}
t^{\prime}-1 \\
j-i-1
\end{array}\right)\left(\begin{array}{c}
t^{\prime}-j+i \\
i
\end{array}\right)+(-1)^{j}\left(\begin{array}{c}
t^{\prime} \\
j
\end{array}\right),
\end{aligned}
$$

by Theorem 2.1. Now we compute $h_{3}\left(\Delta_{t^{\prime}}\left(C_{n}\right)\right)$ :

$$
h_{3}\left(\Delta_{t^{\prime}}\left(C_{n}\right)\right)=n \sum_{i=0}^{2}(-1)^{i}\left(\begin{array}{c}
t^{\prime}-1 \\
2-i
\end{array}\right)\left(\begin{array}{c}
t^{\prime}-3+i \\
i
\end{array}\right)-\left(\begin{array}{l}
t^{\prime} \\
3
\end{array}\right)=-\left(\begin{array}{l}
t^{\prime} \\
3
\end{array}\right)<0,
$$

since $t^{\prime} \geq 3$. Therefore, if $k$ is an infinite field, we obtain that $R / I_{\Delta_{t^{\prime}}\left(C_{n}\right)}$ is not CohenMacaulay by Theorem 5.4. When $k$ is finite, we consider $k[\Delta] \otimes_{k} k(x) \cong k(x)[\Delta]$ in which $x$ is an indeterminate. Then by [3, Theorem 2.1.10] we get the result.

ACKNOWLEDGEMENTS. The first and second authors would like to thank the Institute for Research in Fundamental Sciences (IPM) for financial support. The research of the first author was in part supported by a grant from IPM (No. 93050220). 


\section{REFERENCES}

1. M. Barile and N. Terai, Arithmetical ranks of Stanley-Reisner ideals of simplicial complexes with a cone. Comm. Algebra 38(10) (2010), 3686-3698.

2. P. Brumatti and A.F. da Silva, On the symmetric and Rees algebras of (n,k)-cyclic ideals. 16th School of Algebra, Part II, Brasilia, Brazil, 2000 (Portuguese). Mat. Contemp. 21 (2001), $27-42$.

3. W. Bruns and J. Herzog, Cohen-Macaulay rings. Cambridge Studies in Advanced Mathematics, vol. 39 (Cambridge University Press, Cambridge, UK, 1993).

4. A. Conca and De Negri, M-sequences, graph ideals and ladder ideals of linear type, $J$. Algebra 211(2) (1999), 599-624.

5. J. A. Eagon and V. Reiner, Resolutions of Stanely-Reisner rings and Alexander duality. J. Pure Appl. Algebra 130 (1998), 265-275.

6. H. T. Hà and A. Van Tuyl, Monomial ideals, edge ideals of hypergraphs, and their graded Betti numbers. J. Algebraic Combin. 27(2) (2008), 215-245.

7. H. Haghighi, N. Terai, S. Yassemi and R. Zaare-Nahandi, Sequentially $S_{r}$ simplicial complexes and sequentially $S_{2}$ graphs, Proc. Amer. Math. Soc. 139(6) (2011), 1993-2005.

8. G. Hegedüs, Betti numbers of Stanley-Reisner rings with pure resolutions. arXiv. math.AC/1101.5565v1 (2011).

9. J. Herzog and T. Hibi, Monomial ideals (Springer, New York, NY, 2010).

10. G. Rinaldo, N. Terai and K. Yoshida, On the second powers of Stanley-Reisner ideals, J. Commut. Algebra 3(3) (2011), 405-430.

11. S. Saeedi Madani and D. Kiani, Cohen-Macaulay and Gorenstein path ideals of trees, Algebra Colloq. (to appear).

12. S. Saeedi Madani, D. Kiani and Naoki Terai, Sequentially Cohen-Macaulay path ideals of cycles, Bull. Math. Soc. Sci. Math. Roumanie 54(102) No. 4 (2011), 353-363.

13. N. Terai and N. V. Trung, Cohen-Macaulayness of large powers of Stanley-Reianer ideals. arXiv. math.AC/1009.0833v1 (2010).

14. R. H. Villarreal, Monomial algebras (Marcel Dekker, New York, NY, 2001).

15. K. Yanagawa, Alexander duality for Stanley-Reisner rings and squarefree $\mathbb{N}^{n}$-graded modules, J. Algebra 225 (2000), 630-645. 